\title{
POSTER
}

\section{Le tabac non fumé est-il sans risque pour la muqueuse buccale? À propos d'une observation clinique}

\author{
Radoï L',2, Provost M ${ }^{1,2,3}$, Renoux $M^{1,2}$ \\ Montrouge, France \\ ${ }^{2}$ Hôpital Louis Mourier, service d'odontologie (AP-HP), Colombes, France \\ ${ }^{3}$ Interne en DESCO \\ Introduction
}

${ }^{1}$ Faculté de chirurgie dentaire Paris Descartes, département de médecine buccale et de chirurgie buccale,

Les résultats des études épidémiologiques sur le risque de lésions précancéreuses et de cancers de la cavité buccale liés à la consommation de tabac non fumé (TNF) sont controversés. Le TNF est utilisé de deux façons : prisé et chiqué, sucé ou laissé dans les vestibules jugaux ou labiaux'. L'effet délétère du TNF varie en fonction du processus de fabrication (fermentation du tabac et présence d'adjuvants). II augmente avec la fréquence et la durée de consommation et se manifeste par des kératoses, érythroplasies, fibroses sous-muqueuses et carcinomes épidermoïdes ou verruqueux buccaux'. L'arrêt de l'utilisation du tabac non fumé conduit généralement à la régression des lésions buccales.

\section{Observation}

Un patient âgé de 40 ans, d'origine sri-lankaise, consulte pour des douleurs et des mobilités dentaires au niveau de la région postérieure gauche mandibulaire. II ne présente aucun antécédent médical ou chirurgical et consomme uniquement du TNF (Makla Ifrikia) depuis 25 ans, environ 15 heures quotidiennement, qu'il place toujours au fond du vestibule mandibulaire gauche. Du fait d'un réflexe nauséeux prononcé, l'hygiène buccale du patient est insuffisante. Une adénopathie unique sous-angulo-mandibulaire gauche, mobile, élastique, non douloureuse, est présente. L'examen clinique et radiologique dentaire met en évidence une maladie parodontale généralisée, avec une alvéolyse quasi-terminale au niveau des molaires mandibulaires gauches. L'examen des muqueuses montre une lésion érythro-leucoplasique au niveau du fond du vestibule mandibulaire gauche, du repli jugal et du rempart alvéolaire, à l'endroit où le patient plaçait le tabac. L'examen anatomopathologique de la biopsie de la lésion muqueuse a montré un aspect histologique de leucoplasie inflammatoire et un foyer de dysplasie de bas grade. Informé du risque de transformation maligne de cette lésion pré-néoplasique, le patient a arrêté la consommation. Un assainissement global de la cavité buccale a été réalisé (soins parodontaux et conservateurs, avulsions). Le suivi régulier a permis de constater la disparition de l'adénopathie et la normalisation de la muqueuse buccale après 10 mois. 


\section{Discussion}

Le TNF utilisé dans les pays occidentaux (snus, chewing tobacco) contient du tabac non fermenté recevant un traitement thermique qui détruit les micro-organismes et diminue la libération de nitrates et nitrosamines, cancérogènes. Ce procédé expliquerait le risque de cancer buccal plus faible chez les utilisateurs de ce type de TNF ${ }^{1}$. En revanche, les produits utilisés en Asie et Afrique (shammah, paan, toombak...) sont obtenus en mélangeant le tabac avec des produits nocifs (feuilles de bétel, noix d'Arec, chaux, cendres, épices, condiments), notamment par leur effet caustique 1 .

Le TNF que le patient consommait, le Makla Ifrikia (Shammah ou Yemeni snuff), est un mélange de poudre de tabac, de chaux, de cendres, de poivre noir, d'huiles et d'arômes, ayant un pH très alcalin (>10), responsable de lésions pré-malignes et malignes, avec un relation dose-effet, même chez les patients non-fumeurs et non-buveurs ${ }^{2,3}$. Des récessions gingivales associées aux lésions leucoplasiques sont décrites dans la littérature ${ }^{3}$, comme chez ce patient.

\section{Conclusion}

Des informations sur les dangers du TNF doivent être intégrées dans les programmes d'éducation à la santé bucco-dentaire afin d'éviter son usage et d'aider les utilisateurs à arrêter sa consommation.

\section{Références}

1. International Agency for Research on Cancer. IARC Monographs on Evaluation of Carcinogenic Risk to Humans. Smokeless tobacco and some Tobacco-specific N-Nitrosamines. Volume 87. Lyon, IARC Press 2007.

2. Scheifele $C$ et al. Prevalence of oral cancer and potentially malignant lesions among shammah users in Yemen. Oral Oncol 2007; 43: 42-50.

3. Zhang X et al. Shammah-induced oral leukoplakia-like lesions. Oral Oncol 2001; 37: 609-12. 